\title{
Um Estudo de Autômatos Celulares para o Espalhamento Geográfico de Epidemias com Parâmetro Fuzzy ${ }^{1}$
}

M.S. PEIXOTO2 2 L.C. BARROS 3 , Departamento de Matemática Aplicada, IMECC, UNICAMP, 13083-859 Campinas, SP, Brasil.

\begin{abstract}
Resumo. O modelo SIR (Suscetíveis-Infectados-Recuperados) clássico não considera explicitamente a dimensão espacial de transmissão da doença e assume que os indivíduos têm mesma chance de encontros entre si. Emmendorfer e Rodrigues [2] consideraram vizinhança local e efeitos não locais na evolução da doença, supondo que os contatos são aleatórios. Neste trabalho, o objetivo fundamental é utilizar sistemas fuzzy, baseados em regras lingüísticas, para incorporar os efeitos não locais e um modelo Autômato Celular para estudar o espalhamento geográfico da doença. Simulações numéricas foram realizadas e comparadas com aquelas obtidas por Emmendorfer e Rodrigues [2].
\end{abstract}

\section{Introdução}

A epidemiologia consiste em estabelecer, a partir de observações do fenômeno epidêmico, hipóteses matemáticas para quantificar os conhecimentos biológicos a respeito da dinâmica de transmissão de infecções, para estudar a evolução de epidemias.

Em doença de transmissão direta, que trataremos neste trabalho, para que uma nova infecção ocorra, é necessário que haja indivíduos infectantes e suscetíveis na população para, então, propiciar condições favoráveis para transmissão do agente infeccioso.

A teoria clássica de epidemiologia, em geral, não considera a dimensão espacial de transmissão da doença. Em vez disso, assume que todo indivíduo tem a mesma chance de encontro e, portanto, indivíduos infecciosos têm mesma chance de infectar suscetíveis [1]. Levando-se em conta que a proximidade entre indivíduos seja relevante para um novo contágio, deve-se considerar no modelo, além da variável tempo, também a espacial. Modelos espaciais são indispensáveis para se estudar o espalhamento de uma doença, observando-se o padrão espacial de prevalência da mesma além de revelar a velocidade da frente de onda da epidemia.

\footnotetext{
${ }^{1}$ Trabalho apresentado no XXVI CNMAC.

${ }^{2}$ magda@ime.unicamp.br; Doutoranda com bolsa CNPq.

${ }^{3}$ laeciocb@ime.unicamp.br; orientador.
} 
Emmendorfer e Rodrigues [2] propuseram um modelo de Autômatos Celulares (AC) para estudar a evolução de uma doença de transmissão direta do tipo SIR, levando em conta a estrutura espacial. Considerou dois tipos de proximidades: vizinhança local, que inclui os oito indivíduos geometricamente mais próximos e contatos não locais, considerados aleatórios, que podem ocorrer entre indivíduos que se encontrem a uma distância $L$ :

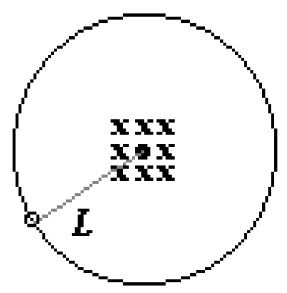

Figura 1: Contatos local, com os oito vizinhos, e não local com algum indivíduo a uma distância $L$ do reticulado.

Em suas simulações, Emmendorfer e Rodrigues [2] escolheram o valor de $L$ de maneira aleatória.

Nossa proposta aqui é estudar os efeitos não locais utilizando Teoria Fuzzy. Mais precisamente, que $L$ seja um parâmetro fuzzy em vez de aleatório como feito em Emmendorfer e Rodrigues [2].

Temos então como objetivos: 1. Estudar um modelo autômato celular para o avanço de epidemias, incluindo aspectos de incertezas; 2. Utilizar controladores fuzzy para modelar o parâmetro L, que representa a distância de um contato não local .

\section{Descrição do Modelo SIR a ser estudado}

O estudo de epidemias através de modelos matemáticos tem sido feito há quase um século. Dentre os modelos mais comuns para representar a dinâmica de epidemias devemos destacar o modelo compartimental SIR (Suscetíveis-InfectadosRecuperados) de Kermack e McKendrick [4] representado pelo diagrama na Figura 2 .

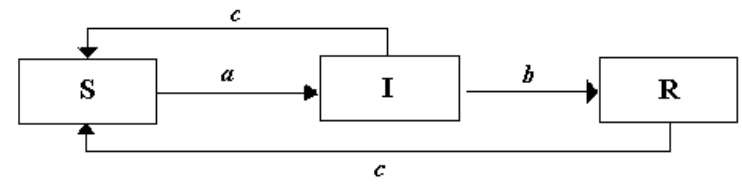

Figura 2: Modelo Compartimental SIR

A equações que representam o modelo SIR são:

$$
\left\{\begin{array}{l}
\frac{d S}{d t}=-a S I+c(I+R) \\
\frac{d I}{d t}=a S I-b I-c I \\
\frac{d R}{d t}=b I-c R
\end{array}\right.
$$


onde $S, I$ e $R$ representam respectivamente as populações de suscetíveis, infectados e recuperados, $a$ é o coeficiente de transmissão, $b$ é o coeficiente de recuperação e $c$ é o coeficiente de mortalidade e de natalidade. Assume-se aqui que indivíduos recuperados estão imunes, e os infectados são automaticamente infectantes.

Para os modelos epidemiológicos clássicos, um parâmetro essencial é o valor de reprodutividade basal, $R_{0}$, que dá o número de casos secundários causados por um indivíduo infectado introduzido numa população totalmente suscetível. Esse parâmetro indica em que condições a doença se propaga na população: se um indivíduo infectado consegue provocar mais que um novo caso, $R_{0}>1$, a doença se propaga. Por outro lado, quando $R_{0}<1$, então a doença se extingue. Este parâmetro tem grande importância na epidemiologia, pois o esforço de se controlar ou erradicar uma doença está intimamente relacionado a ele. Em se tratando de epidemiologia matemática, o valor de reprodutividade basal é essencialmente uma grandeza matemática, sendo que o seu valor pode ser derivado da força de infecção [7]. Desta forma é de se esperar que $R_{0}$ influencie $L$, já que o controle de uma doença, e conseqüentemente seu alcance, estão ligados ao valor de $R_{0}$.

Para estudar o espalhamento geográfico da epidemia adotaremos um modelo Autômato Celular, a exemplo do que fizeram Emmendorfer e Rodrigues [2].

\section{Estudo e Simulação do Modelo SIR por meio de Autômatos Celulares}

\subsection{Autômatos Celulares}

Os Autômatos Celulares (AC) foram introduzidos nos anos 50 pelo matemático John von Neumann, levando em conta sugestões de Stanislaw Ulam, numa tentativa de modelar processos naturais de auto-reprodução.

Os AC consistem de simulações discretas no tempo, espaço e no estado do sistema. A idéia básica destes modelos consiste em considerar cada posição (ou região) do domínio espacial como sendo uma célula, à qual é atribuído um estado. O estado de cada célula é modificado de acordo com o seu estado e o de suas vizinhas na etapa de tempo anterior, através de uma série de regras simples que tentam imitar as leis

físicas ou biológicas, no nosso caso, que regem o sistema [3].

A principal característica dos AC é a facilidade com que podem ser implementados em virtude da simplicidade de sua formulação e o surpreendente retorno visual capaz de sugerir equilíbrios, órbitas, padrões complexos e estruturas organizadas como formações de ondas, entre outras.

O objetivo final dos modelos AC é uma descrição do comportamento macroscópico do fenômeno e não uma descrição exata e fiel do processo microscópico. Não são, em geral, instrumentos de previsão, devendo ser abordados como um meio de experimentação. Os AC são vistos, não como substitutos dos modelos matemáticos tradicionais, mas como um primeiro passo na formulação destes modelos. Os resultados obtidos através da simulação via autômatos celulares podem confirmar hipóteses para uma posterior formulação de um modelo formal.

Emmendorfer e Rodrigues [2] utilizaram um modelo AC para estudar o avanço 
de epidemia em um modelo do tipo SIR. Nesta abordagem, as variáveis de estado do sistema, assim como o tempo, são discretos. O sistema é representado espacialmente através de um reticulado de células que interagem obedecendo a algumas regras de mudança de estado. A dinâmica do sistema como um todo depende desta interação local entre as células. Cada célula representa um indivíduo, que pode estar em um entre três estados: suscetível $(S)$, infectado $(I)$ e recuperado $(R)$.

Emmendorfer e Rodrigues [2] supuseram que a chance de que um indivíduo suscetível se torne infectado vai depender do número de contatos que ele estabelece com outros indivíduos no intervalo de tempo adotado e também da probabilidade de que cada contato resulte em transmissão. A chance com que cada indivíduo se recupere da doença é também levada em conta.

Outra hipótese é que a população é constante. Por isso quando um indivíduo morre, considera-se que nasce um suscetível na mesma célula que ele ocupava. Também a população é representada como sendo uniformemente distribuída no espaço.

Para os contatos não locais, $p_{n l}$ foi considerada como a probabilidade de que um contato não local seja feito com algum indivíduo que esteja a uma distância $L$ no reticulado (Figura 1).

\subsection{Formulação do Modelo Fuzzy}

É de se esperar que o contato não local a uma distância $L$ dependa da chance de haver o deslocamento, $p_{n l}$, e também do quanto a doença é capaz de evoluir naquele ambiente $\left(R_{0}\right)$. Deste modo, assumimos que $L=L\left(R_{0}, p_{n l}\right)$. Porém, uma expressão analítica de $L$ em função de $R_{0}$ e $p_{n l}$ é praticamente impossível. O que dispomos são de informações incertas do tipo: "quanto maior é o valor de $R_{0}$, maior será o $L "$. Assim sendo, optamos por modelar $L$ por meio de um sistema de base de regras fuzzy, com o intuito de traduzir o conhecimento que se tem sobre a dependência com respeito a $R_{0}$ e $p_{n l}$.

O marco inicial da teoria fuzzy foi o artigo [8] publicado em 1965, pelo matemático Lotfi Asker Zadeh, professor no departamento de engenharia elétrica e ciências da computação da Universidade da Califórnia, em Berkeley, com a principal intenção de dar um tratamento matemático a certos termos lingüísticos subjetivos. Esse seria o primeiro passo no sentido de se programar e armazenar conceitos vagos em computadores, tornando possível a produção de cálculos com informações imprecisas. A idéia de Zadeh foi flexibilizar a pertinência de elementos aos conjuntos criando a noção de grau de pertinência. Um elemento poderia pertencer parcialmente a um dado conjunto. Para modelar matematicamente o tal "conjunto", Zadeh propôs o conceito de conjunto fuzzy.

\subsubsection{Sistema baseado em Regras Fuzzy}

Um sistema baseado em regras fuzzy possui quatro componentes: um processador de entrada (ou fuzzificador), um conjunto de regras lingüísticas, um método de inferência fuzzy e um processador de saída (ou defuzzificador), gerando um número real como saída [6]. 


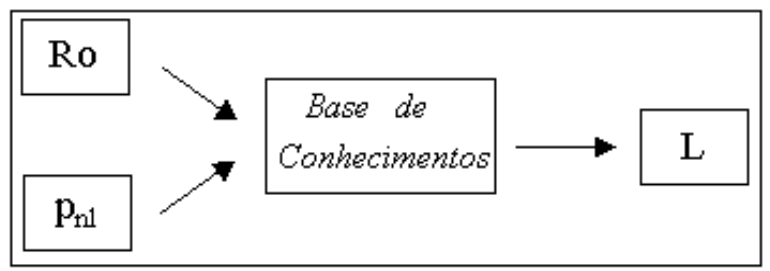

Figura 3: Sistema baseado em regras fuzzy.

No esquema representado na Figura 3, a base de conhecimentos é traduzida por um conjunto de regras fuzzy as quais desempenham o papel de uma função matemática. Com o auxílo de especialistas da área de medicina, obtivemos a seguinte base de regras fuzzy:

1. If (Ro is muitobaixa) and (pnl is muitobaixa) then ( $L$ is pequena)

2. If (Ro is muitobaixa) and (pnl is baixa) then ( $L$ is pequena)

3. If (Ro is muitobaixa) and (pnl is media) then ( $L$ is pequena)

4. If (Ro is muitobaixa) and ( $p n l$ is alta) then ( $L$ is media)

5. If (Ro is muitobaixa) and ( $p n l$ is muitoalta) then ( $L$ is media)

6. If (Ro is baixa) and (pnl is muitobaixa) then ( $L$ is pequena)

7. If (Ro is baixa) and (pnl is baixa) then ( $L$ is pequena)

8. If (Ro is baixa) and (pnl is media) then ( $L$ is media)

9. If (Ro is baixa) and (pnl is alta) then ( $L$ is media)

10. If (Ro is baixa) and (pnl is muitoalta) then ( $L$ is grande)

11. If (Ro is media) and (pnl is muitobaixa) then ( $L$ is pequena)

12. If (Ro is media) and (pnl is baixa) then ( $L$ is pequena)

13. If (Ro is media) and (pnl is media) then ( $L$ is media)

14. If (Ro is media) and (pnl is alta) then ( $L$ is grande)

15. If (Ro is media) and (pnl is muitoalta) then ( $L$ is grande)

16. If (Ro is alta) and (pnl is muitobaixa) then ( $L$ is pequena)

17. If (Ro is alta) and (pnl is baixa) then ( $L$ is media)

18. If (Ro is alta) and (pnl is media) then ( $L$ is media)

19. If (Ro is alta) and (pnl is alta) then ( $L$ is grande)

20. If (Ro is alta) and (pnl is muitoalta) then ( $L$ is grande)

21. If (Ro is muitoalta) and (pnl is muitobaixa) then ( $L$ is media)

22. If (Ro is muitoalta) and (pnl is baixa) then ( $L$ is media)

23. If (Ro is muitoalta) and ( $p n l$ is media) then ( $L$ is grande)

24. If (Ro is muitoalta) and ( $p n l$ is alta) then ( $L$ is grande)

25. If (Ro is muitoalta) and (pnl is muitoalta) then ( $L$ is grande)

Agora cada um dos adjetivos baixa, média, alta, são modelados matematicamente por um conjunto fuzzy através de sua função de pertinência $(u)$ que também foi obtida junto a especialistas. Para o nosso caso, as funções de pertinência são do tipo trapezoidais (Figura 4). 

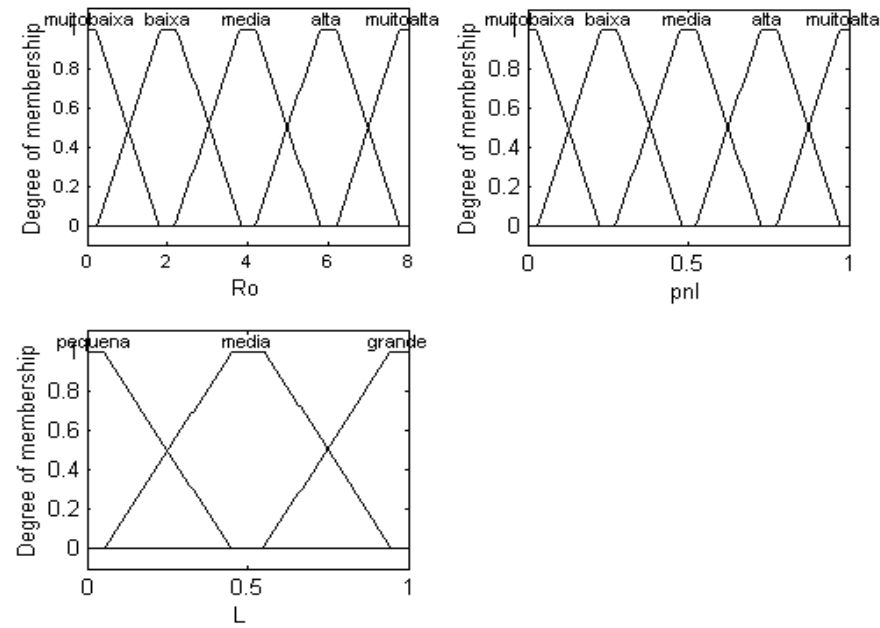

Figura 4: $R_{0}$ e $p_{n l}$ são as variáveis de entrada, $L$ é a variável de saída.

O Método de Inferência de Mamdani [5] agrega as regras através do operador lógico OU, que é modelado pelo operador matemático $(\vee)$ e, em cada regra, os operadores lógicos E e ENTÃO são modelados pelo operador mínimo $(\wedge)$. Para ilustrar o método vamos usar apenas duas regras genéricas, do tipo daquelas que aparecem em nossa base de regras, cada uma com duas entradas e uma saída (Figura $5)$.

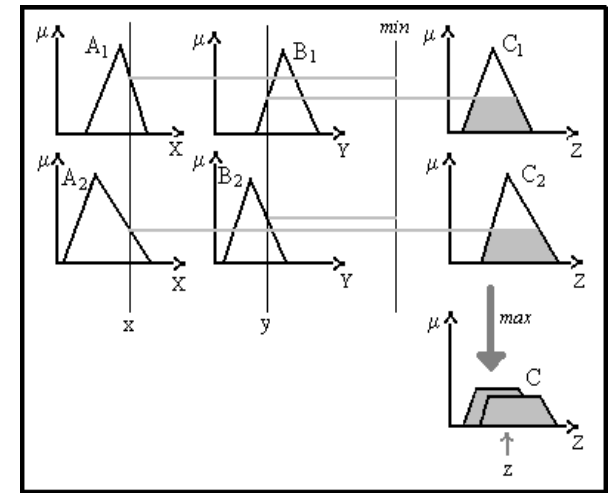

Figura 5: Método de Inferência de Mamdani

A saída geral, $\bar{z}$, do método é dada pela defuzzificação da saída fuzzy obtida a partir dos operadores lógicos descritos acima. No nosso caso usamos a defuzzificação do Centro de Gravidade dado por

$$
\bar{z}=\frac{\int z u(z) d z}{\int u(z) d z}
$$

e obtemos a seguinte superfície como solução do sistema: 


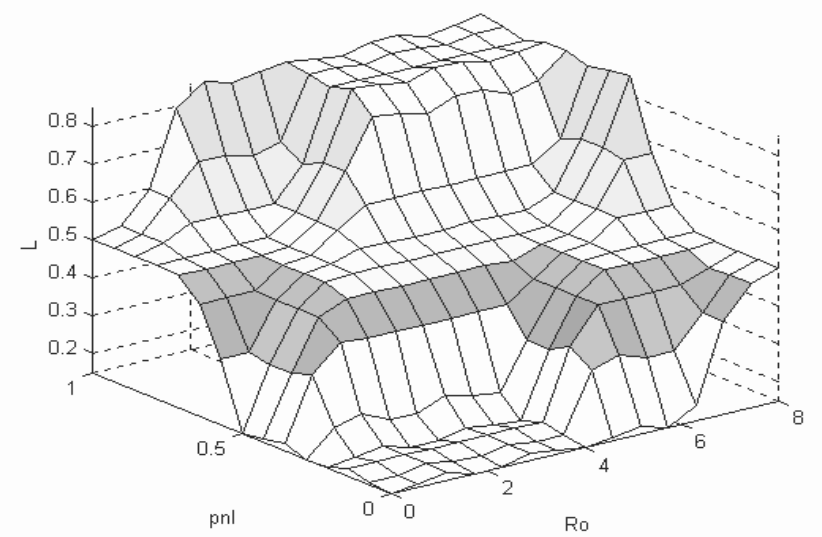

Figura 6: $L=L\left(R_{0}, p_{n l}\right)$

\section{Resultados}

Nas simulações realizadas procuramos observar o avanço de um pequeno foco de uma doença sobre uma população de suscetíveis. Iniciamos as simulações com apenas um infectado no centro do reticulado, e os demais são suscetíveis, como na Figura 1. O indivíduo infectado indicado com a bola cheia está em permanente contato com seus oito vizinhos mais próximos (assinalados com x). No contato não local, é feito um sorteio entre todas as possíveis células na circunferência, para que se determine qual delas será alvo do contato.

Para realizar as simulações numéricas, adotamos os mesmos valores considerados em [2] para que a comparação entre nosso modelo com parâmetro fuzzy e o modelo lá desenvolvido possa ocorrer. Enquanto o valor de $R_{0}$ varia, usamos sempre uma probabilidade pequena de contato não local $\left(p_{n l}=0,1\right)$. Quando dizemos que $R_{0}=0$, devemos assumir que também $L=0$. Notamos que mesmo com essa probabilidade pequena, é possível observar que os contatos não locais exercem um papel fundamental no espalhamento da epidemia.

A evolução dos contingentes populacionais ao longo do tempo para o modelo fuzzy está representada nas Figuras 7 e 8.

Nos dois gráficos das Figuras 7 e 8 , onde $L$ é diferente de zero $\left(p_{n l} \neq 0\right)$, ocorre a formação de focos secundários. À medida que estes focos crescem, eles vão fundindose com a colônia principal.

Em seu trabalho, Emmendorfer e Rodrigues [2] escolheram aleatoriamente os valores de $L$, em particular fez simulações para $L=0,10,20$, que significam raio nulo, médio e grande, respectivamente, com $R_{0}$ e $p_{n l}$ fixos.

Aqui temos as mesmas conclusões por ele obtidas, para valores de mesma grandeza. Porém, no nosso caso, $L$ é um parâmetro obtido por técnicas da teoria fuzzy com interpretação epidemiológica, já que depende de uma grandeza característica da doença em questão $\left(R_{0}\right)$. 


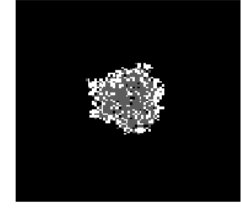

(a)

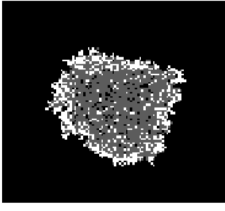

(b)

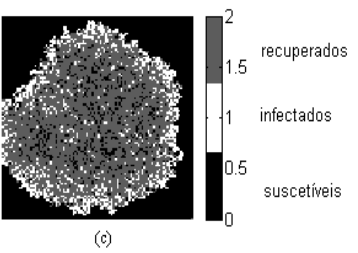

Figura 7: Exemplo de padrões espaciais obtidos através de simulações numéricas no modelo fuzzy para diferentes valores de $R_{0}$ e $p_{n l}$, sendo (a) $R_{0}=5$ e $p_{n l}=0$, (b) $R_{0}=4$ e $p_{n l}=0,1$ e (c) $R_{0}=6$ e $p_{n l}=0,1$.
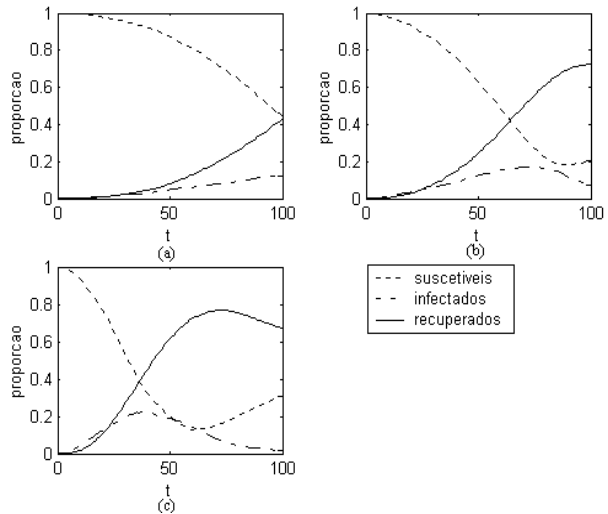

Figura 8: Evolução dos contigentes populacionais ao longo do tempo do modelo fuzzy para diferentes valores de $R_{0}$ e $p_{n l}$, sendo $R_{0}=5$ e $p_{n l}=0, R_{0}=4$ e $p_{n l}=0,1$ e $R_{0}=6$ e $p_{n l}=0,1$, respectivamente.

\section{Agradecimentos}

Ao Prof. Rodney C. Bassanezzi (IMECC/UNICAMP) pela cooperação para o desenvolvimento deste trabalho.

Abstract. The classic model SIR (Susceptible-Infectaed-Recovered) does not consider explicitly the space dimension of disease transmission and assumes that the individuals have same possibility of meeting between themselves. Emmendorfer e Rodrigues [2] considered local neighborhood and non local effect in the evolution of the disease, assuming that the contacts are random. In this work, the main objective is to use a rule-based fuzzy system to incorporate the non local effect and 
a Cellular Automata model to study the geographic scattering of the disease. Numerical simulations were made and compared with those obtained by Emmendorfer e Rodrigues [2].

\section{Referências}

[1] L. Edelstein-Keshet, "Mathematical Models in Biology", McGraw-Hill, Inc, 1987.

[2] L.R. Emmendorfer e L.A.D. Rodrigues, Um modelo de Autômatos Celulares para o Espalhamento Geográfico de Epidemias, Tendências em Matemática Aplicada e Computacional, 2 (2001), 73-80.

[3] G.B. Ermentrout e L. Edelstein-Keshet, Cellular Automata Approaches to Biological Modeling, Theor. Biol., 160 (1993), 97-133.

[4] W.O. Kermack e A.G. McKendrick, A contribution to the mathematical theory of epidemics, Proceedings of the Royal Society of London Series A, 15 (1927), $700-721$.

[5] W. Pedrycz e F. Gomide, "An Introduction to Fuzzy Sets: Analysis and Design", Massachusetts Institute of Technology, 1998.

[6] F. Ribacionka, "Sistemas Computacionais baseados em Lógica Fuzzy", Dissertação de Mestrado, Universidade Mackenzie, São Paulo, SP, 1999. (disponível em http://sites.uol.com.br/fribacio)

[7] H.M. Yang, "Epidemiologia Matemática: estudo dos efeitos da vacinação em doenças de transmissão direta", Editora da Unicamp, 2001.

[8] L.A. Zadeh, Fuzzy Sets, Informat. Control, 8 (1965), 338-353. 
